\title{
Corrigendum: The microbiome of New World
}

\section{vultures}

Michael Roggenbuck, Ida Bærholm Schnell, Nikolaj Blom, Jacob Bælum, Mads Frost Bertelsen,

Thomas Sicheritz-Pontén, Søren Johannes Sørensen, M. Thomas P. Gilbert, Gary R. Graves \& Lars H. Hansen

Nature Communications 5:5498 doi: 10.1038/ncomms6498 (2014); Published 25 Nov 2014; Updated 21 Oct 2015

The original version of this Article contained an error in the spelling of the author Thomas Sicheritz-Pontén, which was incorrectly given as Thomas Sicheritz Pontén. This has now been corrected in both the PDF and HTML versions of the Article. 